\title{
Construction of the noncommutative rank I Bergman domain
}

\author{
Zhituo Wang
}

\begin{abstract}
In this paper we present a harmonic oscillator realization of the most degenerate discrete series representations of the $S U(2,1)$ group and the deformation quantization of the coset space $D=S U(2,1) / U(2)$ with the method of coherent state quantization. This short article is based on a talk given at the 9-th International Workshop, Varna "Lie Theory and Its Applications in Physics" (LT-9).
\end{abstract}

\section{Introduction}

It is believed that ordinary differential geometry should be replaced by noncommutative geometry [1] when we are approaching the Planck scale and quantum field theories defined on noncommutative space time (NCQFT) [2, 3, 4] are considered as the right way to explore the effects of quantum gravity.

The simpliest noncommutative space is the Moyal space, which is a symplectic manifold generated by the noncommutative coordinates $x_{\mu}$, such that $\left[x_{\mu}, x_{\mu}\right]=$ $i \theta_{\mu v}$, where $\theta_{\mu v}$ is a constant. The first well defined quantum field theory on 4 dimensional Moyal space is the Grosse-Wulkenhaar model [5]. It is not only perturbative renormalisable to all orders but also asymptotically safe, namely the beta function for the coupling constant is zero at the fixed point of this model. Hence this model is a candidate to be constructed nonperturbatively, namely it's possible to obtain the exact Green's function which is unique and analytic in the coupling constant, by resumming the perturbation series [6]. Recently the two dimensional Grosse-Wulkenhaar model has been constructed in [7].

Since the noncommutative quantum fields theories are better behaved than their commutative counterparts, it is very natural to construct other noncommutative manifolds and physics models over them.

Laboratoire de Physique Théorique, CNRS UMR 8627,

Université Paris XI, F-91405 Orsay Cedex, France, e-mail: zhituo.wangeth.u-psud.fr 
In this paper we construct the noncommutative coset space $D=S U(2,1) / S(U(2) \times$ $U(1)$ ), with the method of coherent state quantization. For doing this we also introduce a harmonic oscillator realization of the most degenerate discrete series representation of the group $S U(2,1)$ which is a generalization for the $S U(1,1)$ case introduced by $\mathrm{H}$. Grosse and P. Presnajder [13]. The interested reader could look at [13, 12] for more details about the coherent state quantization and [10, 11, 17] for more details about the representation theory of noncompact Lie group. In [8] and [9] we have studied the harmonic oscillator realization of the maximal degenerate discrete series representations for an arbitrary $S U(m, n)$ group.

The construction of the noncommutative coset space $S U(2,1) / U(2)$ has been also studied by [14], [15] with the method of Berezin-Toeplitz quantization and by by [16] with the method of "WKB quantization". The interested reader could go to the references for details.

\section{The $S U(m, 1)$ group and its Lie algebra}

The group $G=S U(m, 1)$ is defined as a subgroup of the matrix group $S L(m+n, C)$ :

$$
g=\left(\begin{array}{cc}
a_{m \times m} & b_{m \times 1} \\
c_{1 \times m} & d
\end{array}\right) \in G
$$

satisfies the constraint

$$
g^{\dagger} \Gamma g=g \Gamma g^{\dagger}=\Gamma, \quad \Gamma=\left(\begin{array}{cc}
I_{m \times m} & 0 \\
0 & -1
\end{array}\right) .
$$

Here I's represents unit matrices and 0's the blocks of zeros.

The maximal compact subgroup is defined by matrices

$$
K=S(U(m) \times U(1))=\left\{\left(\begin{array}{cc}
K_{1} & 0 \\
0 & K_{2}
\end{array}\right), \operatorname{det}\left(K_{1} K_{2}\right)=1\right\} .
$$

The Bergman domain is defined as the coset space $D=G / K$ :

$$
D=\left\{\left.Z|1-| Z\right|^{2}>0\right\}=\left\{\left.z|1-| z_{1}\right|^{2}-\left|z_{2}\right|-\cdots-\left|z_{m}\right|^{2}>0\right\},
$$

where $Z=\left(z_{1}, \cdots, z_{m}\right)$ are the coordinates of the coset space $D$. It is a pseudoconvex domain over which we could define a holomorphic Hilbert spaces [14] with the reproducing Bergman kernel:

$$
K\left(W^{\dagger}, Z\right)=\left(1-W^{\dagger} Z\right)^{-N},
$$

where $Z$ and $W$ are complex m-columns, and $N=m+1, m+2, \cdots$ is a natural number characterizing the representation. 
$D$ is also a Kähler manifold with the Kähler metric defined by the derivations of the Bergman kernel:

$$
g_{i \bar{j}}=\frac{1}{N} \partial_{\bar{z}^{i}} \partial_{z^{j}} \log K\left(Z^{\dagger}, Z\right) .
$$

More explicitly we have:

$$
g_{i \bar{j}}=\left[\frac{\delta_{i j}}{1-|Z|^{2}}+\frac{z_{i} \bar{z}_{j}}{\left(1-|Z|^{2}\right)^{2}}\right], g^{i \bar{j}}=\left(1-|Z|^{2}\right)\left(\delta_{i j}-\bar{z}_{i} z_{j}\right) .
$$

We could easily calculate that the Ricci tensor: $R_{i \bar{j}}=-(m+1) g_{i \bar{j}}$ and the curvature $R=-(m+1)$ and verify that the metric $g_{i j}$ is a solution to the Einstein's equation in the vacuum:

$$
R_{i \bar{j}}-\frac{1}{2} g_{i \bar{j}} R+\Lambda g_{i \bar{j}}=0
$$

with the cosmological constant $\Lambda=\frac{m+1}{2}$.

The Lie algebra $\mathbf{g}=\operatorname{Lie}(G)=\operatorname{su}(m, 1)$ is defined by $M=\left\{\left(\begin{array}{cc}A & B \\ B^{\dagger} & D\end{array}\right)\right\} \in \mathbf{g}$, $M^{\dagger} \Gamma=-\Gamma M$. where $A^{\dagger}=-A, D^{\dagger}=-D, \operatorname{tr}(A+D)=0$.

Consider the Cartan decomposition of the Lie algebra $\mathbf{g}=\mathbf{l}+\mathbf{p}$ and let $\mathbf{a} \in \mathbf{p}$ be a maximal Abelian subalgebra. We could choose for a the set of all matrices of the form

$$
H_{t}=\left(\begin{array}{ccc}
O_{(m-1) \times(m-1)} & O_{(m-1) \times 1} & O_{(m-1) \times 1} \\
O_{1 \times(m-1)} & 0 & t \\
O_{1 \times(m-1)} & t & 0
\end{array}\right)
$$

where $t$ is a real number.

Define the linear functional over $H_{t}$ by $\alpha\left(H_{t}\right)=t$, the roots of $(\mathbf{g}, \mathbf{a})$ are given by

$$
\pm \alpha, \pm 2 \alpha,
$$

with multiplicities $m_{\alpha}=2$ and $m_{2 \alpha}=1$.

Define

$$
\delta:=\left\{\mathbf{a}_{t} \mid \mathbf{a}_{t}=\exp H_{t}, H_{t} \in \mathbf{a}\right\} .
$$

so we have

$$
\mathbf{a}_{t}=\left(\begin{array}{ccc}
I & O & 0 \\
O & \cosh t & \sinh t \\
0 & \sinh t & \cosh t
\end{array}\right),
$$

where the symbol $I$ stands for the identity matrix and $O$ is the matrix with entries 0 . 


\section{The holomorphic discrete series of representations of the $S U(m, 1)$ group}

The unitary irreducible representations for $G=S U(m, 1)$ are the principal series, the discrete series and the supplementary series. We consider only the discrete series of representations, which are realized in the Hilbert space $\mathscr{L}_{N}^{2}(D)$ of holomorphic functions with the the inner product defined by:

$$
(f, g)_{N}=\int d \mu_{N}(Z, \bar{Z}) \bar{f}(\bar{Z}) g(Z)
$$

where $d \mu_{N}(Z, \bar{Z})=c_{N}\left[\operatorname{det}\left(E-Z^{\dagger} Z\right)\right]^{N-(m+1)}|d Z|$ is the normalised measure and $c_{N}=\pi^{-2}(N-2)(N-1)$.

The discrete series of representations $T_{N}$ is defined by

$$
T_{N} f(Z)=[\operatorname{det}(C Z+d)]^{-N} f\left(Z^{\prime}\right), N=m+1, m+2, \cdots
$$

where

$$
Z^{\prime}=(A Z+B)(C Z+d)^{-1}
$$

In the following we consider only the $m=2$ case and construct the harmonic oscillator realization of the most degenerate discrete series representation (14). We introduce a $3 \times 1$ matrix $\hat{Z}=\left(\hat{z}_{a}\right), a=1,2,3$, of bosonic oscillators acting in Fock space and satisfying commutation relations

$$
\begin{gathered}
{\left[\hat{z}_{a}, \hat{z}_{b}^{\dagger}\right]=\Gamma_{a b}, a, b=1,2,3} \\
{\left[\hat{z}_{a}, \hat{z}_{b}\right]=\left[\hat{z}_{a}^{\dagger}, \hat{z}_{b}^{\dagger}\right]=0,}
\end{gathered}
$$

where $\Gamma$ is a $3 \times 3$ matrix defined in (2). It can be easily seen that for all $g \in S U(2,1)$ these commutation relations are invariant under transformations:

$$
\hat{Z} \mapsto g \hat{Z}, \quad \hat{Z}^{\dagger} \mapsto \hat{Z}^{\dagger} g^{\dagger}
$$

We could define the creation and anhilation operators $\hat{a}_{\alpha}$ and $\hat{b}$ as:

$$
\hat{Z}=\left(\begin{array}{c}
\hat{a} \\
\hat{b}^{\dagger}
\end{array}\right):\left[\hat{a}_{\alpha}, \hat{a}_{\beta}^{\dagger}\right]=\delta_{\alpha \beta}, \alpha, \beta=1,2 .\left[\hat{b}, \hat{b}^{\dagger}\right]=1,
$$

and all other commutation relations among oscillator operators vanish.

The Fock space $\mathscr{F}$ in question is generated from a normalized vacuum state $|0\rangle$, satisfying $\hat{a}_{\alpha}|0\rangle=\hat{b}|0\rangle=0$, by repeated actions of creation operators:

$$
\left|m_{\alpha}, n\right\rangle=\prod_{\alpha} \frac{\left(\hat{a}_{\alpha}^{\dagger}\right)^{m_{\alpha}}\left(\hat{b}^{\dagger}\right)^{n}}{\sqrt{m_{\alpha} ! n !}}|0\rangle
$$


We shall use the terminology that the state $\left|m_{\alpha}, n\right\rangle$ contains $m=\sum m_{\alpha}$ particles $a$ and $n$ particles $b$.

Consider a basis of $s u(2,1)$ Lie algebra $X=X_{a b}^{A} A=1, \cdots 8, a, b=1,2,3$, we assign the operator

$$
\hat{X}=-\hat{Z}^{\dagger} \Gamma X \hat{Z}=-\hat{z}_{a}^{\dagger} \Gamma_{a b}^{A} X_{a b}^{A} \hat{z}_{b} .
$$

Their anti-hermicity follows directly:

$$
\hat{X}^{\dagger}=-\operatorname{tr}\left(\hat{Z}^{\dagger} X^{\dagger} \Gamma \hat{Z}\right)=+\operatorname{tr}\left(\hat{Z}^{\dagger} \Gamma X \hat{Z}\right)=-\hat{X} .
$$

Using commutation relations for annihilation and creation operators we have:

$$
[\hat{X}, \hat{Y}]=\left[\hat{Z}^{\dagger} \Gamma X \hat{Z}, \hat{Z}^{\dagger} \Gamma Y \hat{Z}\right]=-\hat{Z}^{\dagger} \Gamma[X, Y] \hat{Z} .
$$

So that the operators $\hat{X}_{a}$ satisfy in Fock space the $s u(2,1)$ commutation relations. The assignment

$$
g=e^{\xi^{A} X_{A}} \in S U(2,1) \Rightarrow \hat{T}(g)=e^{\xi^{A} \hat{X}_{A}}
$$

then defines a unitary $S U(2,1)$ representation in Fock space.

\section{The Coherent States Quantization of $D=S U(2,1) / U(2)$ and the Star Product}

We briefly describe the construction of coherent states on coset space of a Lie group following [12]. Let $T_{g}$ be an unitary irreducible representation of an arbitrary Lie group $G$ in a Hilbert space $\mathscr{H},\left|z_{0}\right\rangle \in \mathscr{H}$ is a normalized state in the Garding space of $T_{g}$. Let $K$ be the stability group of the $\left|z_{0}\right\rangle$, for which $T_{k}\left|z_{0}\right\rangle=e^{i \alpha(k)}\left|z_{0}\right\rangle$, for $k \in K$. Then for each $z=g_{z} z_{0} \in D=G / K$ we could assign a coherent states $:|z\rangle=\psi_{z}=$ $T\left(g_{z}\right)\left|z_{0}\right\rangle$. Define the functions $\omega_{0}(g)=<z_{0}|T(g)| z_{0}>$ and $\omega(g, z)=\left\langle z\left|T_{g}\right| z\right\rangle=$ $\omega_{0}\left(g_{z}^{-1} g g_{z}\right)$. As $\left|z_{0}\right\rangle$ is in the Garding space, $\omega(g)$ is a smooth function in $g$.

For $G=S U(2,1)$, the state $\left|z_{0}\right\rangle$ is defined in the Fock space as:

$$
\left.\left|z_{0}\right\rangle=\frac{\left(\hat{b}^{\dagger}\right)^{N}}{\sqrt{(N) !}}\left|0>=\frac{1}{\sqrt{N}}\right| 0,0 ; N\right\rangle .
$$

Here $N$ is a natural number that specifies the representation: $\hat{N}\left|z_{0}\right\rangle=N\left|z_{0}\right\rangle$. All other states in the representation space are obtained by the action of rising operators given in 20). The stability group for $\left|z_{0}\right\rangle$ is $K=S(U(2) \times U(1))$.

Using the $K \delta K$ decomposition of $g=k^{\dagger} \delta q$ [17], for which $k, q \in K$, we obtain:

$$
\omega_{0}(g)=\left\langle z_{0}|\hat{T}(g)| z_{0}\right\rangle=\frac{1}{\cosh t}\left[(1+\ln \cosh t) e^{i(\alpha(q)-\alpha(k))}\right]^{N}
$$

Consider an operator acting on $\mathscr{H}$ : 


$$
\hat{F}=\int d g \tilde{F}(g) T(g)=\int d g \tilde{F}(g) \omega(g, z)
$$

where $\tilde{F}(g)$ ia a distribution on a group $G$ with compact support. We also define for each $\hat{F}$ a biholomorphic function:

$$
F(z, \bar{z})=\left\langle\Psi_{z}|\hat{F}| \Psi_{z}\right\rangle
$$

The star product of two such bi-holomorphic functions $F$ and $G$ is then defined by [13]:

$$
\begin{aligned}
& (F \star G)(z, \bar{z})=\left\langle\Psi_{z}|\hat{F} \hat{G}| \Psi_{z}\right\rangle=(F \star G)(z, \bar{z}) \\
= & \int d g_{1} d g_{2} \tilde{F}\left(g_{1}\right) \tilde{F}\left(g_{2}\right) \omega\left(g_{1} g_{2}, z\right) .
\end{aligned}
$$

Obviously the star product defined above is noncommutative, associative and is invariant under the action of the group $G$. The noncommutative algebra of functions $\{F(z)\}$ induces a noncommutative structure on the coset space $D$. That's how we construct the noncommutative version of the Bergman domain, which is noted as $\hat{D}$.

Now we shall study the explicit form of the star product for $G=S U(2,1)$. Using the explicit form of the group element $g=e^{\xi^{A} X_{A}}$ and integration by parts we have:

$$
F_{A_{1} \ldots A_{n}}(z)=\left.(-1)^{n}\left(\partial_{\xi_{A_{1}}} \ldots \partial_{\xi_{A_{n}}} \omega\right)\left(e^{\xi^{A} \hat{X}_{A}} z\right)\right|_{\xi=0}=(-1)^{n}\left\langle z\left|\hat{X}_{A_{1}} \ldots \hat{X}_{A_{n}}\right| z\right\rangle .
$$

Here $\hat{X}_{A},(A=1 \cdots, 8)$ are the left-invariant vector field on group $G$ corresponding to the Lie algebra basis $X_{A}$ whose explicit form is given in [9].

From the definition of the star product $(28)$ it follows that:

$$
\begin{gathered}
\left(F_{A_{1} \ldots A_{n}} \star F_{B_{1} \ldots B_{m}}\right)(z) \\
=\left.(-1)^{n+m}\left(\hat{X}_{A_{n}} \ldots \hat{X}_{A_{1}} \hat{X}_{B_{m}} \ldots \hat{X}_{B_{1}} \omega\right)(g, z)\right|_{g=e} .
\end{gathered}
$$

We define the function $\xi_{A}$ as the expectation value of the operator $\hat{X}_{A}$ between the coherent states as:

$$
\xi_{A}(z)=\frac{1}{N}\left\langle z\left|\hat{X}_{A}\right| z\right\rangle=\frac{1}{N}\left\langle z_{0}\left|\hat{T}^{\dagger}\left(g_{z}\right) \hat{X}_{A} \hat{T}\left(g_{z}\right)\right| z_{0}\right\rangle .
$$

The star product between these coordinates functions reads:

$$
\left(\xi_{A} \star \xi_{B}\right)(z)=\frac{1}{N^{2}}\left\langle z\left|\hat{X}_{A} \hat{X}_{B}\right| z\right\rangle=\left(1+A_{N}\right) \xi_{A}(z) \xi_{B}(z)+\frac{1}{2 N} f_{A, B}^{C} \xi_{C}(z)+B_{N} \delta_{A, B},
$$

where $A_{N}$ and $B_{N}$ depend on the Bernoulli numbers coming from the BakerCampbell-Hausdorff formula and are of order $1 / N$. We see that the parameter of the non-commutativity is $\lambda_{N}=1 / N$. For $N \rightarrow \infty$ we recover the commutative product. 
According to the Harish-Chandra imbedding theorem, we could always imbed the commutative maximal Hermitian symmetric space into the noncompact part of the Cartan subalgebra. So the coordinates of the noncommutative Bergman domain $\hat{D}$ can be identified as the coordinate functions corresponding to the noncompact Cartan subalgebra.

\section{Conclusions and prospectives}

In this paper we have constructed the noncommutative Bergman domain $\hat{D}$ whose commutative counterpart is the coset space $D=G / K$, where $G=S U(2,1)$ and $K=$ $S(U(2) \times U(1))$. This result could be generalized to an arbitrary type one rank one Cartan domain $D=G=S U(m, 1) / S(U(m) \times U(1))$ straightforwardly.

In [9] we have build a model of quantum theory of real scalar fields on the noncommutative manifold $\hat{D}$ and find that the one loop quantum correction to the 2 point function is finite. This is a hint of the finiteness of quantum field theory on $\hat{D}$ and this deserves further studies.

Ackonledgements The author is very grteful to Harald Grosse and Peter Presnajder for useful discussions.

\section{References}

1. A. Connes, "Noncommutative geometry" Academic Press, Boston, MA, 1994.

2. M. R. Douglas and N. A. Nekrasov, "Noncommutative field theory," Rev. Mod. Phys. $\mathbf{7 3}$ (2001) 977 [hep-th/0106048].

3. R. J. Szabo, "Quantum field theory on noncommutative spaces," Phys. Rept. 378 (2003) 207 hep-th/0109162

4. V. Rivasseau, "Non-commutative Renormalization," arXiv:0705.0705 [hep-th]. Séminaire Bourbaphy.

5. H. Grosse and R. Wulkenhaar, "Renormalisation of phi**4 theory on noncommutative $\mathrm{R} * * 4$ in the matrix base," Commun. Math. Phys. 256, 305 (2005) |arXiv:hep-th/0401128].

6. V. Rivasseau, From Perturbative to Constructive Renormalization, Princeton University Press, 1991.

7. Zhituo Wang, "Construction of 2-dimensional Grosse-Wulkenhaar Model," arXiv:1104.3750 [math-ph].

8. H. Grosse, P. Presnajder and Zhituo Wang, "Quantum Field Theory on quantized Bergman domain,” J. Math. Phys. 53 (2012) 013508 [arXiv:1005.5723 [math-ph]].

9. Zhituo Wang, "Deformation quantization of rank I Bergman domain," arXiv:1110.2632 [math$\mathrm{ph}$.

10. W. Rudin, Function theory in the unit ball of $C^{n}$, Springer-Verlag, 1980.

11. A. A. Kirillov, Elements of theory of representations, Springer-Verlag, Berlin, 1976; Representation Theory and Noncommutative Harmonic Analysis II, Encyklopedia of Math. Sciences 59, Springer-Verlag, Berlin, 1995.

12. A. M. Perelomov, Generalized coherent states and their applications, Springer-Verlag, Berlin, 1986.

13. H. Grosse and P. Prešnajder, The construction of noncommutative manifolds using coherent states, Let. Math. Phys. 28 (1993) 239-250. 
14. D. Bortwick, A. Lesniewski and H. Upmeier, Non-perturbative deformation quantization of Cartan Domains, J. Functional Analysis 113 (1993) 153-176; H. Upmeier, Toeplitz operators and index theory in several complex variavbles, Harmonic Analysis 1996, Birkhäuser, 1996.

15. G. Jakimowicz and A. Odziewicz, Quantum complex Minkowski space, J. of Geom. and Phys. 56 (2006) 1576-1599.

16. P. Bieliavsky, R. Gurau, V. Rivasseau, "Non Commutative Field Theory on Rank One Symmetric Spaces," J. Noncommut. Geom. 3 (2009) 99-123. [arXiv:0806.4255] [hep-th]].

17. Anthony K. Knapp, Lie group: beyond an introduction- 2nd ed., Birkhauser Boston, Basel, Berlin, 2002

18. M. Rieffel, Lie Group convolutions algebras as deformation quantization of linear Poisson structures, Amer. J. Math. 112 (1989) 137-216. 